\title{
NOTE
}

\section{A New Screening Approach for Glycolipid-type Biosurfactant Producers Using MALDI-TOF/MS}

\author{
Shun Sato ${ }^{1}$, Tokuma Fukuoka ${ }^{1}$, Azusa Saika', Tatsuyuki Koshiyama ${ }^{2}$, and \\ Tomotake Morita ${ }^{1 *}$
${ }^{1}$ Research Institute for Sustainable Chemistry, National Institute of Advanced Industrial Science and Technology (AIST), Tsukuba Central 5-2, 1-1-1 Higashi, Tsukuba, Ibaraki 305-8565, JAPAN Fukushima Pref. 974-8686, JAPAN \\ ${ }^{2}$ Agrochemical Research Department, Organic Synthesis Research Laboratories, Kureha Corporation, 16 Ochiai, Nishiki-Machi, Iwaki,
}

\begin{abstract}
We applied matrix-assisted laser desorption/ionization time-of-flight mass spectrometry (MALDITOF/MS) to screen for glycolipid-type biosurfactant (BS) producers. A crude extract of Pseudozyma antarctica, a well-known mannosylerythritol lipid (MEL) producer, was initially subjected to MALDI-TOF/ MS. The spectrum of the extract showed the accumulation of diacylated MELs in culture. We then screened 80 environmental samples for BS-producing yeasts, and extracts from broth cultures of the selected five strains were examined using MALDI-TOF/MS. The results showed that all five strains produced MELs, whereas four strains also produced cellobiose lipids (CLs). By D1/D2 region sequence analysis, the MELproducing strain was assigned to $P$. antarctica while the four MEL- and CL-producing strains were assigned to $P$. hubeiensis. These results demonstrate that MALDI-TOF/MS is a rapid and reliable tool to detect BS molecules in crude extracts of broth cultures to screen for glycolipid-type BS producers.
\end{abstract}

Key words: biosurfactant, MALDI-TOF/MS, Pseudozyma, screening

\section{Introduction}

Some environmental microbes are known to produce extracellular amphiphilic compounds called biosurfactants (BSs). Among these compounds, glycolipid-type BSs are a major class of BSs produced by a kind of microbes, including prokaryotes and eukaryotes. Mannosylerythritol lipids (MELs, Fig. 1A), cellobiose lipids (CLs, Fig. 1B), rhamnolipids, sophorolipids, and trehalose lipids are well-known glycolipid-type $\mathrm{BSs}^{1,2)}$. Their structural variations depend on the producer: Pseudozyma antarctica produces mainly MELs, Ustilago maydis produces mainly CLs, Pseudomonas aeruginosa produces rhamnolipids, Starmerella bombicola produces sophorolipids, and Rhodococcus sp. produces trehalose lipids ${ }^{3)}$. Glycolipid-type BSs can be used in industrial products because they possess complex bulky structures and chiral carbons, leading to unique self-assembling abilities, high surface-active properties, and biological activities ${ }^{3-5}$. These properties have expanded the potential applications of BSs in the fields of detergents, cosmetics, foods, and pharmaceutics. Their biodegradability and biocompatibility have also attracted attention to the utilization of BSs as alternatives to conventional synthetic surfactants to build a sustainable society. However, few examples of commercial BS production have been presented because numerous producers secrete small amounts of BS in culture with limited structural variation.

The isolation of new microbes with the ability to produce large amounts of BS is necessary for the large-scale production of BSs. Several studies have screened for superior BS producers ${ }^{6-9)}$. In general, screening for BS producers includes: (i) the enrichment of microbes from select microbial sources, (ii) evaluation of the products in microbial cultures based on their surface-active properties, and(iii) structural determination of the BSs. Of these steps, structural determination requires a large amount of product purified through time-consuming methods such as column chromatography. Since the BS titers in broth cultures are usually low, scale-up cultivation is required to prepare samples for structural analysis. This is also time-consuming and prevents efficient screening for BS producers. Thus, to

\footnotetext{
* Correspondence to: Tomotake Morita, Research Institute for Sustainable Chemistry, National Institute of Advanced Industrial Science and Technology (AIST), Tsukuba Central 5-2, 1-1-1 Higashi, Tsukuba, Ibaraki 305-8565, JAPAN

E-mail: morita-tomotake@aist.go.jp

Accepted September 23, 2019 (received for review July 1, 2019)

Journal of Oleo Science ISSN 1345-8957 print / ISSN 1347-3352 online

http://www.jstage.jst.go.jp/browse/jos/ http://mc.manusriptcentral.com/jjocs
} 
(A)

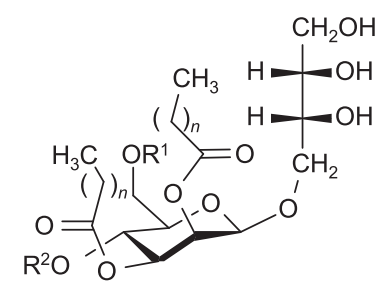

$\mathrm{R}^{1}=\mathrm{R}^{2}=\mathrm{H}$ or Ac, $n=6-10$
(B)

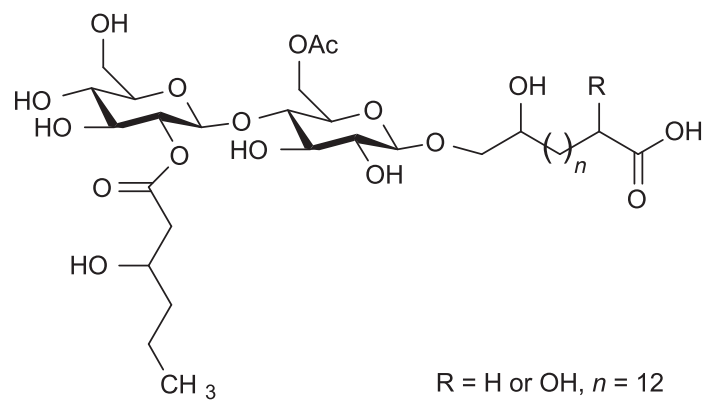

Fig. 1 Chemical structures of BSs. (A) MELs and (B) CLs.

enable rapid and efficient screening for BS producers, it is preferable to determine the structures of BSs using crude samples during the early stages of the screening process.

Matrix-assisted laser desorption/ionization time-of-flight mass spectrometry (MALDI-TOF/MS) is commonly used to identify organic molecules with a high molecular weight (MW) and complex structures based on precise mass-tocharge ratio $(\mathrm{m} / \mathrm{z})$ measurements. MALDI-TOF/MS requires a small amount of sample for measurement and accepts mixtures because its precise measurement of the $\mathrm{m} / \mathrm{z}$, corresponding to the MWs of the analytes, can distinguish structural differences. Recent studies have shown the structural confirmation of purified glycolipid-type BSs using this analytical method ${ }^{10-13)}$. However, the capability of MALDI-TOF/MS to screen for BS producers directly using crude extracts has not been explored. Because the variations among BSs depend on the microbes that produce them, structural information about the BSs in crude products obtained by MALDI-TOF/MS could provide taxonomic information, accelerating the screening process for BS producers.

In this study, we applied MALDI-TOF/MS to detect BSs in crude culture extracts. The method was used to identify MELs in crude samples, and then it was used to screen for BS producers from environmental samples. We identified two Pseudozyma species as MEL producers using this method, supporting the applicability of MALDI-TOF/MS as a rapid and reliable screening method for BS producers.

\section{Experimental}

\subsection{Preparation of crude extracts from culture broth con-} taining MELs

Pseudozyma antarctica JCM10317 was used as an authentic MEL producer. MEL production was performed at $25^{\circ} \mathrm{C}$ in a 5 -L jar fermenter (B. E. Marubishi, Tokyo, Japan) containing $2.0 \mathrm{~L}$ of a MEL production medium composed of $0.3 \%(\mathrm{w} / \mathrm{v}) \mathrm{NaNO}_{3}, 0.1 \%(\mathrm{w} / \mathrm{v})$ yeast extract, $0.03 \%(\mathrm{w} / \mathrm{v})$ $\mathrm{KH}_{2} \mathrm{PO}_{4}$, and $0.03 \%(\mathrm{w} / \mathrm{v}) \mathrm{MgSO}_{4} \cdot 7 \mathrm{H}_{2} \mathrm{O}$. The agitation speed was set at $500 \mathrm{rpm}$ and the air flow rate was $1.0 \mathrm{~L} / \mathrm{min}$. A total of $40 \mathrm{~mL}$ of soybean oil were fed at the beginning of the cultivation and a $20 \mathrm{~mL}$ portion of soybean oil was fed on days 5 and 7 of cultivation. After 12 days of cultivation, the culture broth was extracted with an equal volume of ethyl acetate, followed by thin-layer chromatography (TLC) and MALDI-TOF/MS.

\subsection{Analytical methods}

TLC was performed on a Merck F60 glass plate with a solvent system of $\mathrm{CHCl}_{3} / \mathrm{CH}_{3} \mathrm{OH} / 12 \% \mathrm{NH}_{3}$ aq. $=65 / 15 / 2$ (by vol.). The spots on the plates were visualized by spraying with $2 \%(\mathrm{w} / \mathrm{v})$ anthrone- $\mathrm{H}_{2} \mathrm{SO}_{4}$ reagent, followed by heating at $110^{\circ} \mathrm{C}$ for 5 min. Purified MELs (containing MEL-A, -B, and $-\mathrm{C}$ ) were used as the standard. After evaporation of the ethyl acetate, a crude mixture containing BSs was subjected to MALDI-TOF/MS (JMS-S3000 SpiralTOF; JEOL Ltd., Tokyo, Japan) with 2,4,6-trihydroxyacetophenone as the matrix.

\subsection{Screening for BS-producing yeasts}

A total of 80 samples from the natural environment were obtained in the region of East Japan (Iwate, Miyagi, Fukushima, Ibaraki, Tochigi, Gunma, and Saitama prefectures and Tokyo). They included 48 from leaves, 8 from soils, 7 from mosses, 6 from tree branches, 5 from mushrooms, 3 from fruits, 1 from a root, 1 from a flower, and 1 from a water plant. They were transferred to a test tube containing $2 \mathrm{~mL}$ of basal medium containing $0.3 \%(\mathrm{w} / \mathrm{v}) \mathrm{NH}_{4} \mathrm{NO}_{3}$, $0.1 \%(\mathrm{w} / \mathrm{v})$ yeast extract, $0.03 \%(\mathrm{w} / \mathrm{v}) \mathrm{KH}_{2} \mathrm{PO}_{4}$, and $0.03 \%$ $(\mathrm{w} / \mathrm{v}) \mathrm{MgSO}_{4} \cdot 7 \mathrm{H}_{2} \mathrm{O}$. Soybean oil was added to the medium at $2 \%(\mathrm{v} / \mathrm{v})$ as the sole carbon source. To inhibit bacterial growth, $0.01 \%(\mathrm{w} / \mathrm{v})$ streptomycin sulfate and $0.005 \%(\mathrm{w} /$ v) chloramphenicol were added to the medium. The test tubes were shaken at $25^{\circ} \mathrm{C}$ and 200 strokes/min on a reciprocal shaker(BR-43FL; TAITEC, Saitama, Japan) for 3-5 days until the culture broth became turbid. An aliquot of culture broth after serial dilution was then spread on an agar medium containing the basal medium plus $10 \%(\mathrm{w} / \mathrm{v})$ glucose and the antibiotics. After growth at $25^{\circ} \mathrm{C}$, colonies with different shapes and colors were picked out and stocked on basal agar medium containing $10 \%(\mathrm{w} / \mathrm{v})$ 
glucose for the following experiments.

As the primary screening step, BS accumulation in the culture was estimated based on the drop-collapse method $^{14)}$. The colonies picked were cultured in a deepwell plate (96 wells) containing $1.0 \mathrm{~mL}$ of basal medium plus $10 \%(\mathrm{w} / \mathrm{v})$ glucose, followed by shaking on a deep-well maximizer(TAITEC) at $25^{\circ} \mathrm{C}$ and $1600 \mathrm{r} / \mathrm{min}$ for 2 days. An aliquot $(20 \mu \mathrm{L})$ of culture broth was directly dropped onto a slip of parafilm, after which areas of the drops were visually observed. Cultures showing a wider spread compared with uninoculated culture medium were then extracted with ethyl acetate for TLC.

At the secondary screening step, culture broths from flask cultivations were examined by TLC and MALDI-TOF/ MS. Selected strains were cultured in basal medium plus $10 \%(\mathrm{w} / \mathrm{v})$ glucose and/or $2 \%(\mathrm{v} / \mathrm{v})$ soybean oil at $25^{\circ} \mathrm{C}$ and $200 \mathrm{rpm}$ for up to 14 days. The culture broth was checked by TLC after extraction with ethyl acetate, followed by MALDI-TOF/MS.

\subsection{Ribosomal DNA(rDNA) sequencing and molecular phylogenic analysis}

A genetic analysis of the screened strains was performed by the DNA sequences in D1/D2 region large subunit of 28S rRNA. All DNA sequences were determined by the Fasmac DNA Sequence Service (Atsugi, Japan). Phylogenetic analysis was performed with ClustalW2.1 (http:// clustalw.ddbj.nig.ac.jp/) ${ }^{15)}$ and the obtained phylogenetic tree was drawn using NCBI Tree Viewer(https://www.ncbi. nlm.nih.gov/tools/treeviewer/). All sequences were deposited in the DNA Data Bank of Japan under accession numbers LC488391-LC488395.

\subsection{Chemicals}

All reagents were of analytical grade from Wako Pure Chemicals (Osaka, Japan) and Tokyo Chemical Industries (Tokyo, Japan) and were used as received.

\section{Results}

3.1 Validation of the MALDI-TOF/MS method to detect BSs in crude culture extracts

We initially examined cultures containing MELs to detect expected spectra based on MALDI-TOF/MS. The representative MEL producer $P$. antarctica JCM10317 was cultivated in MEL production medium containing soybean oil, after which the products were extracted with ethyl acetate. The ethyl acetate fraction was confirmed to contain MEL-A as a predominant component with small amounts of MEL-B and MEL-C based on TLC (Fig. 2A). The fraction was then subjected to MALDI-TOF/MS measurement; the obtained spectrum is shown in Fig. 2B. The main peak at an $\mathrm{m} / \mathrm{z}$ of 671.3 was observed in good agreement with the sodium $\operatorname{adduct}\left([\mathrm{M}+\mathrm{Na}]^{+},+22.9 \mathrm{Da}\right)$ of MEL-A (diacetyl type, $\mathrm{MW}=648.2$ ) with acyl chains of total 18 carbon numbers as hydrophobic moieties. We also observed peaks at $\mathrm{m} / \mathrm{z}$ values of 643.3 and 699.4 , which corresponded to sodium
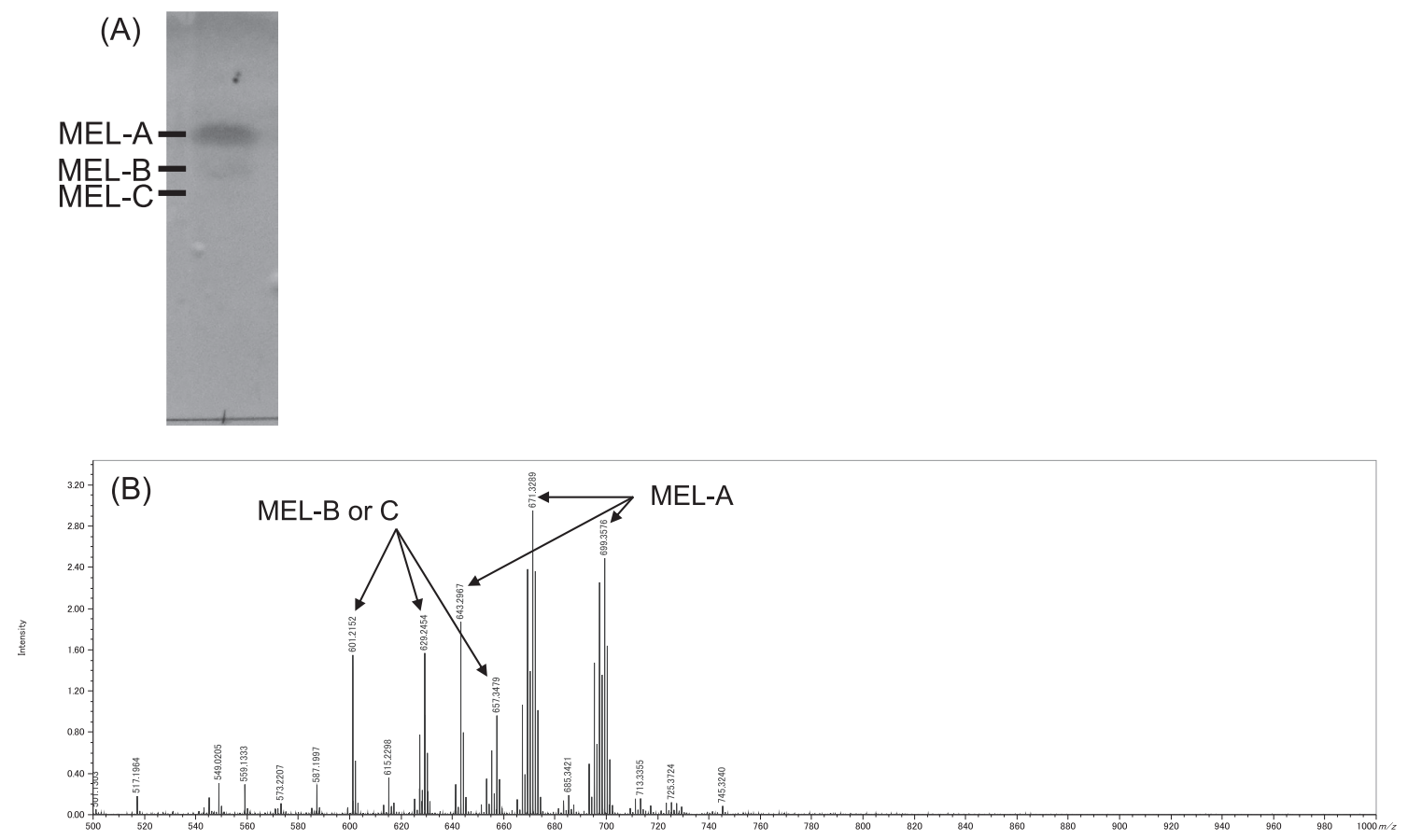

Fig. 2 MEL detection in a crude extract from the culture broth of P. antarctica. (A) TLC analysis and (B) the MALDI-TOF/ MS spectrum. 
adducts of MEL-As with acyl chains of total 16(MW = $620.4)$ and $20(\mathrm{MW}=676.5)$ carbon numbers, respectively. Additionally, the observed peaks at $\mathrm{m} / \mathrm{z}$ values of 601.2 , 629.2 , and 657.3 were in good agreement with sodium adducts of MEL-B or MEL-C, which were detectable by TLC. There were no peaks at $m / z$ values of 800 and higher, in which triacylglycerols are observed. These results demonstrate that BSs in crude extracts from culture broth were clearly detectable based on MALDI-TOF/MS.

\subsection{Primary screening for BS-producing microbes}

To examine MALDI-TOF/MS as an effective method for screening for BS-producers, 80 environmental samples (e.g., leaves, soils, mosses, and branches) were harvested and used as sources for microbial enrichment. After shaking for 3-5 days with $2 \%(\mathrm{v} / \mathrm{v})$ soybean oil under aerobic conditions, 58 samples became turbid. Each culture broth from the 58 samples was then spread onto agar plates and cells forming colonies were selected. Consequently, 181 colonies were picked out and subjected to deep-well plate cultivation to test for BS-producing ability using the drop-collapse method. Of 181 cultures, 51 showed distinct droplets with a wider distribution compared with an uninoculated culture. Using TLC, we examined extracts of the selected 51 cultures, and 8 extracts showed blue spots with $R_{\mathrm{f}}$ values of 0.30 to 0.66 (Fig. S1). This result indicated that these cultures contained amphiphilic compounds with an anthorne-positive structure. Of the 8 samples, 5 cultures (strains 10-1, 32-2, 32-3, 40-1, and 75-3) were selected based on microbial origin and TLC patterns for the next step.

\subsection{MALDI-TOF/MS identification of the products from the selected strains}

The selected five strains were subjected to cultivation in $300 \mathrm{~mL}$ flasks containing $30 \mathrm{~mL}$ of basal medium with soybean oil and/or glucose to elucidate product variations. After extraction with ethyl acetate, glycolipid-type BSs were visualized by TLC before MALDI-TOF/MS measurement. An extract from a culture in which 10-1 grew on glucose showed a clear blue spot corresponding to MELs as well as a slower-moving broad spot, as shown on the TLC plates (Fig. 3). On the contrary, the other four extracts showed multiple spots by TLC in extracts from cultures with glucose. The addition of soybean oil to these cultures produced more hydrophobic compounds than glucose only. Thus, we divided the five cultures into two groups; namely, groups A (10-1) and B (32-2, 32-3, 40-1, and 75-3), and respective representatives (10-1 and 32-3, respectively) were analyzed in detail using MALDI-TOF/MS.

The MS spectrum of an extract from a culture in which 10-1 grew on glucose revealed monoacyl and diacyl MELs with traces of triacylgycerol (Fig. 4A). Table 1 shows peaks in the spectrum corresponding to BSs and their structural features. Signals at $\mathrm{m} / z$ values of $545.3,503.3$, and 461.2 were in good agreement with sodium adducts of monoacyl MEL-A $(M W=522.4)$, $-\mathrm{B}$, or $-\mathrm{C}(\mathrm{MW}=480.4)$ and $-\mathrm{D}($ no acetyl type, $\mathrm{MW}=438.3$ ) with the decyl group as a hydrophobic chain, respectively. Diacyl MELs were observed in the main signals at $m / z=699.5$ and 671.4 , corresponding to MEL-A with acyl chains of total $20(\mathrm{MW}=676.6)$ and 18 $(\mathrm{MW}=648.5)$ carbon numbers, respectively. Small signals corresponding to MWs of MEL-B or -C were also observed. Because of the dominance of monoacyl MELs with a decyl chain, diacyl MELs would also contain decyl chains as a primary hydrophobic component.

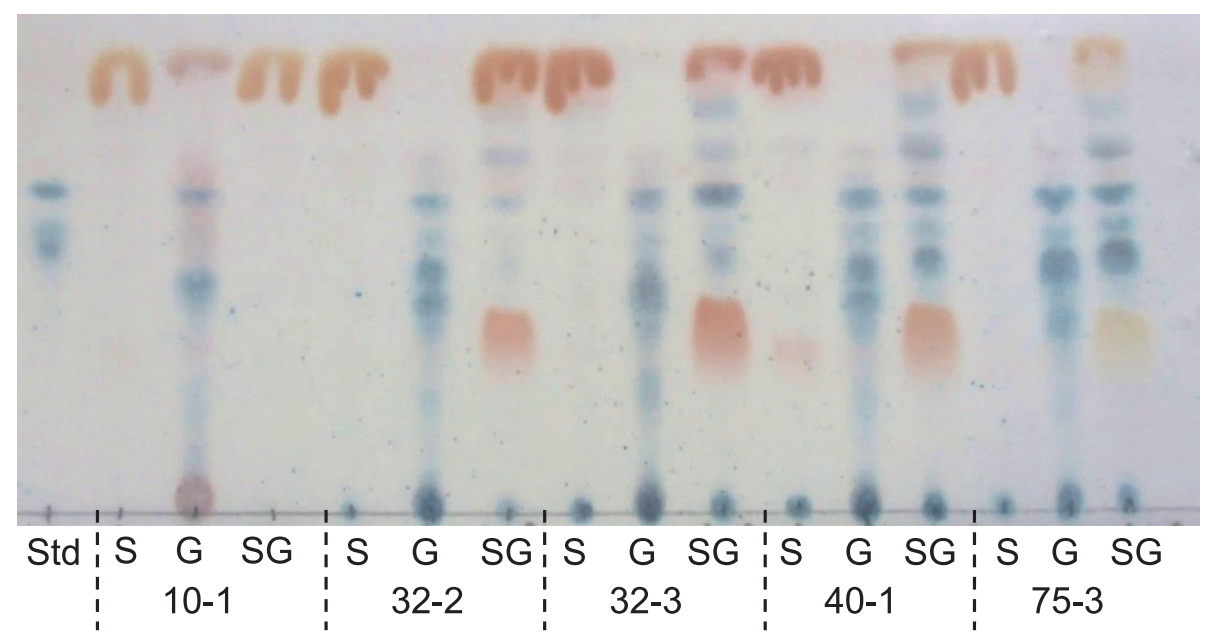

Fig. 3 TLC analysis of extracts from culture broths of five selected strains (10-1, 32-2, 32-3, 40-1, and 75-3). Std, MEL standards containing MEL-A, -B, and -C; S, extracts from soybean oil cultures; G, extracts from glucose cultures; SG, extracts from soybean oil and glucose cultures. 
(A)

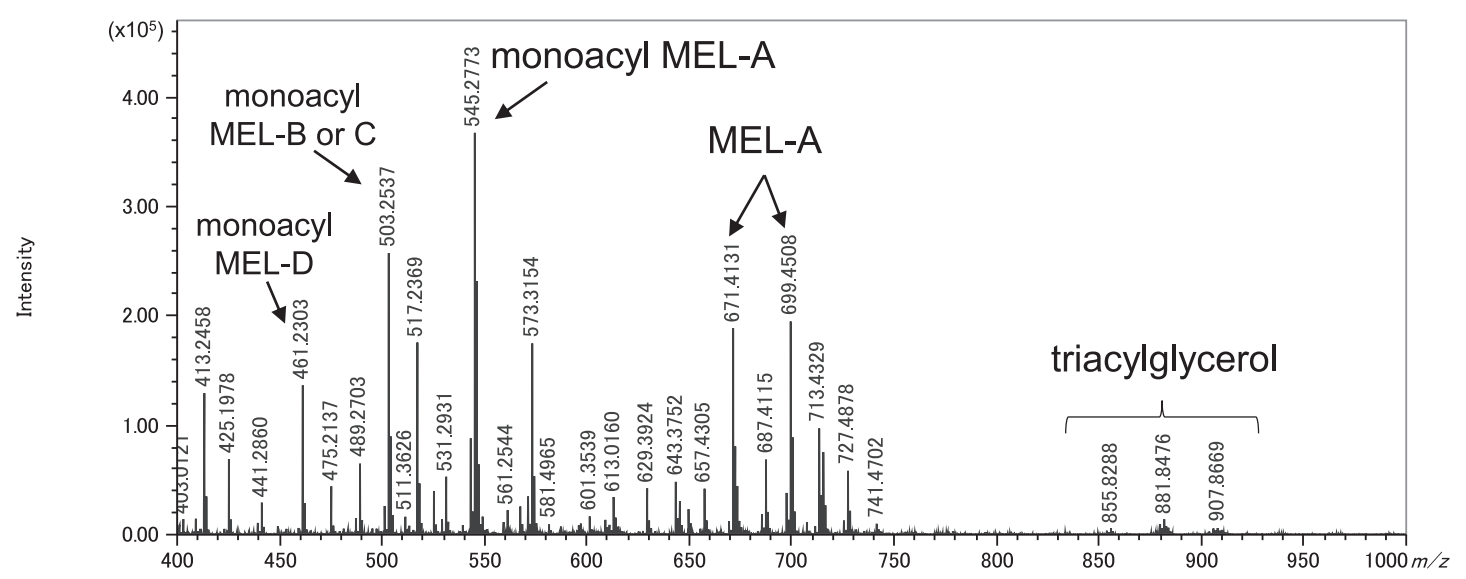

(B)

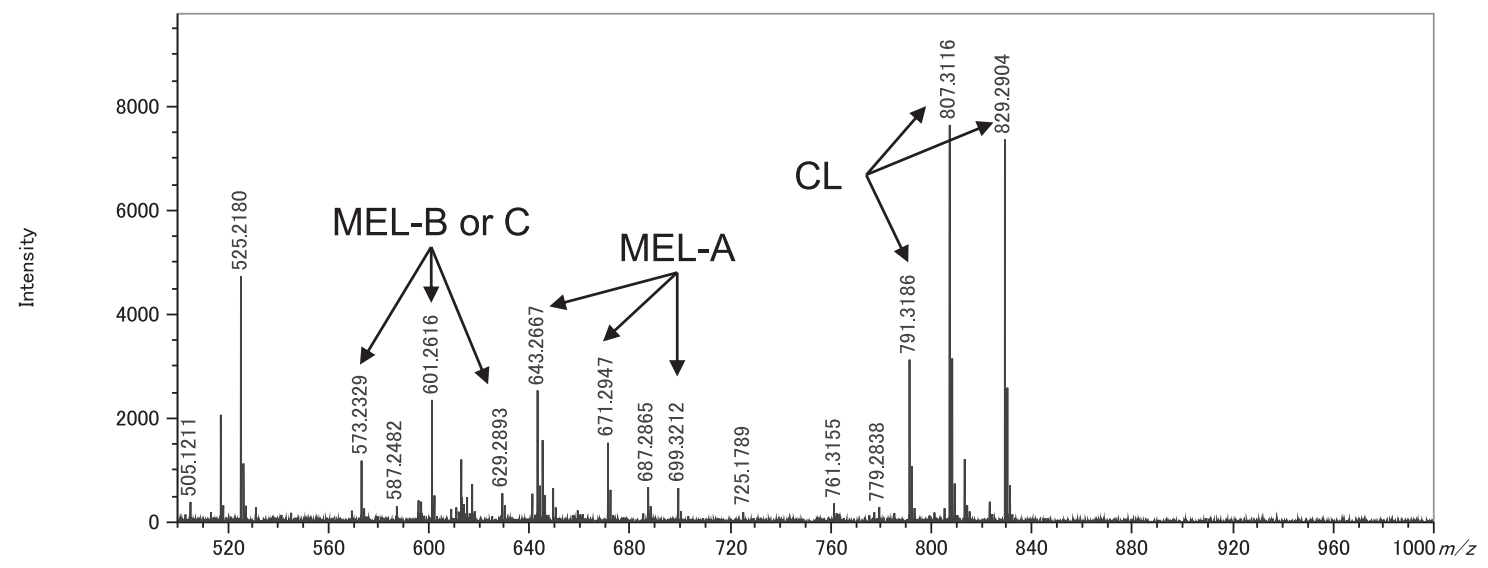

(C)

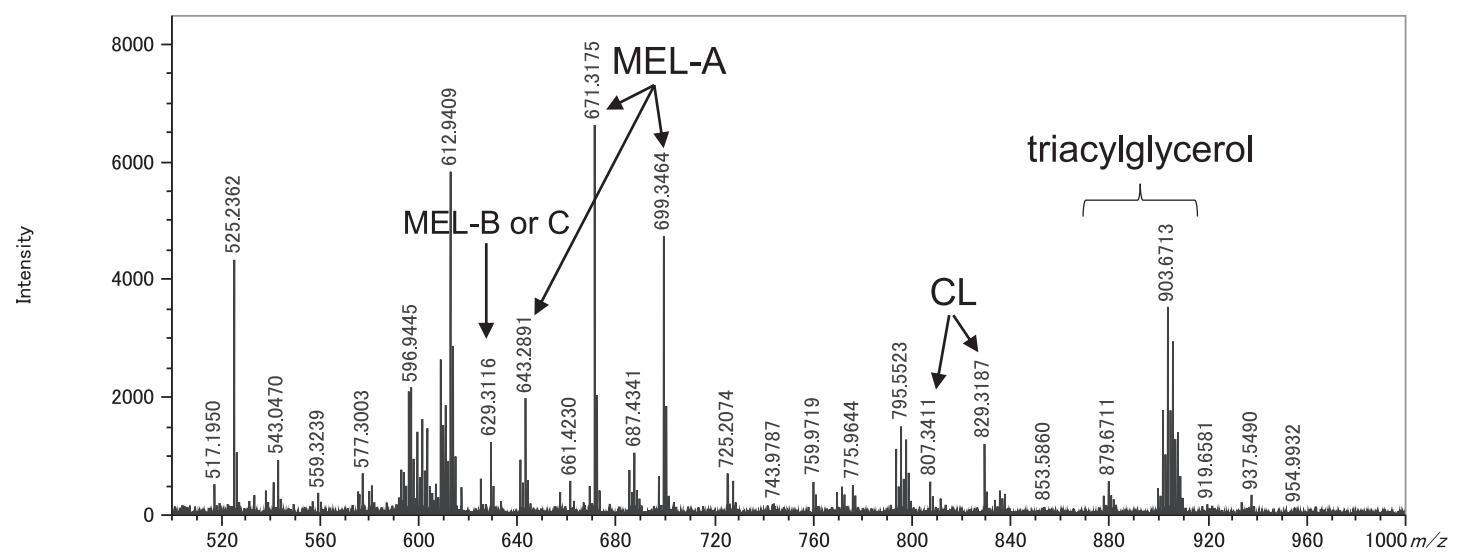

Fig. 4 MALDI-TOF/MS analyses of crude extracts from broth cultured with the selected strains and conditions. (A) 10-1 grown on glucose, (B) 32-3 grown on glucose, (C) 32-3 grown on soybean oil and glucose. 
S. Sato, T. Fukuoka, A. Saika et al.

Table 1 Observed $m / z$ values in MS spectra and the corresponding BS structures.

\begin{tabular}{|c|c|c|c|c|c|}
\hline Strains & $\begin{array}{l}\text { Carbon } \\
\text { sources }\end{array}$ & $\begin{array}{c}m / z \\
\text { observed }\end{array}$ & $\begin{array}{l}\text { Molecular } \\
\text { weight, } \\
\text { estimated }\end{array}$ & Type of BSs & $\begin{array}{c}\text { Sum of carbon numbers } \\
\text { in acyl chains } \\
\text { (except for acetyl) }\end{array}$ \\
\hline \multirow[t]{15}{*}{$10-1$} & Glucose & 461.2 & 438.3 & monoacyl MEL-D & 10 \\
\hline & & 489.3 & 466.4 & monoacyl MEL-D & 12 \\
\hline & & 475.2 & 452.3 & monoacyl MEL-B or -C & 8 \\
\hline & & 503.3 & 480.4 & monoacyl MEL-B or -C & 10 \\
\hline & & 531.3 & 508.4 & monoacyl MEL-B or -C & 12 \\
\hline & & 517.2 & 494.3 & monoacyl MEL-A & 8 \\
\hline & & 545.3 & 522.4 & monoacyl MEL-A & 10 \\
\hline & & 573.3 & 550.4 & monoacyl MEL-A & 12 \\
\hline & & 601.4 & 578.5 & MEL-B or -C & 16 \\
\hline & & 629.4 & 606.5 & MEL-B or -C & 18 \\
\hline & & 657.4 & 634.5 & MEL-B or -C & 20 \\
\hline & & 643.4 & 620.5 & MEL-A & 16 \\
\hline & & 671.4 & 648.5 & MEL-A & 18 \\
\hline & & 699.5 & 676.6 & MEL-A & 20 \\
\hline & & 727.5 & 704.6 & MEL-A & 22 \\
\hline \multirow[t]{14}{*}{$32-3$} & Glucose & 573.2 & 550.3 & MEL-B or -C & 14 \\
\hline & & 601.3 & 578.4 & MEL-B or -C & 16 \\
\hline & & 629.3 & 606.4 & MEL-B or -C & 18 \\
\hline & & 643.3 & 620.4 & MEL-A & 16 \\
\hline & & 671.3 & 648.4 & MEL-A & 18 \\
\hline & & 699.3 & 676.4 & MEL-A & 20 \\
\hline & & 791.3 & 768.4 & CL, monohydroxy & 22 \\
\hline & & 807.3 & 784.4 & CL, dihydroxy & 22 \\
\hline & & 829.3 & 806.4 & CL, dihydroxy, sodium salt & 22 \\
\hline & $\begin{array}{c}\text { Soybean oil } \\
+ \text { glucose }\end{array}$ & 629.3 & 606.4 & MEL-B or $-\mathrm{C}$ & 18 \\
\hline & & 643.3 & 620.4 & MEL-A & 16 \\
\hline & & 671.3 & 648.4 & MEL-A & 18 \\
\hline & & 699.3 & 676.4 & MEL-A & 20 \\
\hline & & 807.3 & 784.4 & CL & 22 \\
\hline
\end{tabular}

On the contrary, the MS spectrum of a crude extract from a culture in which 32-3 grew on glucose revealed the presence of CLs as well as monoacyl and diacyl MELs (Fig. 4B). Strong signals at $m / z=807.3$ and 829.3 , corresponding to MWs of 784.4 and 806.4, respectively, were observed. These values were in good agreement with the structures of CLs with dihydroxy groups in the acyl chain (Fig. 1B) and its sodium salt. In addition, a signal at $m / z=791.3$ was assigned to CLs with the monohydroxy group in the acyl chain. Signals at $m / z=573.2,601.3$, and 629.3 corresponded to MEL-B or -C with acyl chains of total 14, 16, and 18 carbon numbers, respectively, whereas signals at $\mathrm{m} / \mathrm{z}=$ 643.3, 671.3, and 699.3 corresponded to MEL-A with the same acyl components of MEL-B or -C detected. These patterns of MS spectra were observed in crude extracts from other cultures belonging to group B (data not shown). In addition, the MS spectrum of the extract from the culture in which 32-3 grew on glucose and soybean oil showed MELs as the main components (Fig. 4C). Signals at $m / z=671.3$ and 699.3 corresponded to the MWs of MEL-As, and a small signal at $\mathrm{m} / z=629.3$ corresponded to that of MEL-B or -C. A set of small signals corresponding 


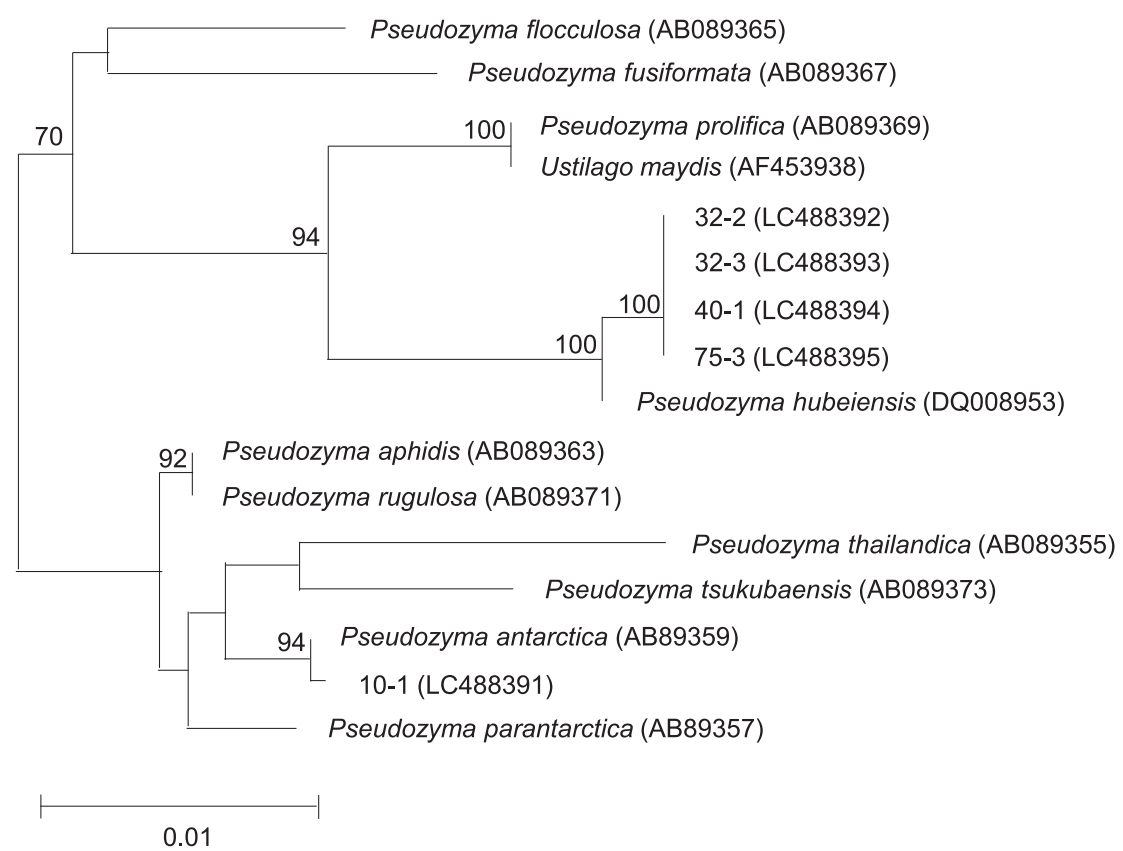

Fig. 5 Phylogenetic relationship of the screened strains and related taxa based on their D1/D2 region sequences. Percentage numbers at the nodes indicate the levels of bootstrap support (a frequency of less than $50 \%$ is not shown)for the brunch point based on 1000 bootstrap replicates. The DDBJ/GenBank/EMBL accession numbers are indicated in parentheses.

to CLs was also detected. MS spectra of extracts from other cultures belonging to group B grown on glucose and soybean oil also showed the same product trend (Fig. S2).

Overall, MALDI-TOF/MS indicated that strain 10-1 belonging to group A produced MELs as glycolipid-type BSs and the other four strains belonging to group B produced MELs and CLs simultaneously.

\subsection{Identification of the selected strains}

The above five strains were examined based on D1/D2 region sequence analysis and taxonomic identification was performed. As shown in Fig. 5, strain 10-1 belonging to group A was identified as $P$. antarctica, whereas the other four strains belonging to group B were identified as $P$. $h u$ beiensis. To evaluate MEL production, strains 10-1, 32-3, and 75-3, identified as $P$. antarctica and two $P$. hubeiensis strains, respectively, were further examined by cultivation in MEL production medium. Under this culture condition, strain 10-1 produced a large amount of MEL-A in culture broth from $4 \%(\mathrm{v} / \mathrm{v})$ soybean oil in 7 days, whereas strains 32-3 and 75-3 produced MEL-C as a dominant product, as revealed by TLC (Fig. S3).

\section{Discussion}

Screening for novel BS-producing microbes is a useful strategy to find candidates for industrial BS producers. However, the complicated structure of glycolipid-type BSs is a barrier to the rapid identification of products in the screening process, even though an analysis method such as nuclear magnetic resonance and MS with isolated samples has been established. Thus, for accelerating the screening process, we subjected crude extracts from cultures directly to MALDI-TOF/MS measurement and identified glycolipidtype BSs.

In crude extracts from cultures of $P$. antarctica, MALDITOF/MS distinguished peaks corresponding to MELs (Fig. 2 ). Differences in the number and chain lengths of acyl moieties were detectable. MELs with different numbers of acyl chain lengths have been identified by gas chromatography-MS after separation by column chromatography followed by methanolysis to obtain fatty acid methylesters; however, by applying MALDI-TOF/MS to directly measure crude samples, structural variations in MELs were identified. This could allow us to rapidly identify the molecular skeletons of BSs. Thus, this methodology would be applicable to distinguish types of BS molecules in crude samples.

Screening for BS producers by applying MALDI-TOF/MS allowed for the identification of products from unidentified microbial cultures (Fig. 4). Based on the results of MALDITOF/MS, we divided five strains into two groups, one producing MELs and the other producing MELs and CLs simultaneously. Previous reports showed that MEL producers could be taxonomically characterized by their MEL productivity and structural variations ${ }^{9,16)}$. The present screening process showed that one strain producing MELs was classified as $P$. antarctica, whereas the other four 
strains producing MELs and CLs were classified as $P$. hubeiensis. We confirmed that strain 10-1 showed high productivity of MEL-A from soybean oil in the presence of $\mathrm{NaNO}_{3}$, whereas strains 32-3 and 75-3 produced MEL-C as the main component under the same cultivation conditions. The species-dependent structural variations in MELs demonstrated here are consistent with previous reports ${ }^{9}$.

Additionally, the application of MALDI-TOF/MS to crude extracts allowed us to detect minor components in cultivation products. In the MS spectra of extracts from cultures in which strain 10-1 grew on glucose, we detected monoacyl MEL-A as a minor constituent (Fig. 4A). This type of MEL was considerable but its presence has not been stated. Therefore, MALDI-TOF/MS could be a useful tool to identify new types of BSs. Thus, the application of MALDITOF/MS to screen for BS producers will enable the rapid and reliable identification of useful strains for BS production and novel BS molecules with advanced properties.

\section{Acknowledgements}

We thank Ms. Wujisiguleng and Mr. hayashibara for their technical assistances.

\section{Supporting Information}

This material is available free of charge via the Internet at http://dx.doi.org/jos.68.10.5650/jos.ess 19177

\section{References}

1) Banat, I.M.; Makkar, R.S.; Cameotra, S.S. Potential commercial applications of microbial surfactants. Appl. Microbiol. Biotechnol. 53, 495-508(2000).

2) Robin, G.; Wim, S.; Inge, V.B. Biotechnological opportunities in biosurfactant production. Curr. Opin. Biotechnol. 30, 66-72(2014).

3) Bruno, N.P.; Marina, G.P.; Mario, C.R.M.; Gustavo, M.; Iramaia, A.N.-N.; Glaucia, M.P. Current status in biotechnological production and applications of glycolipid biosurfactants. Appl. Microbiol. Biotechnol. 100, 10265-10293 (2016).

4) Morita, T.; Fukuoka, T.; Imura, T.; Kitamoto, D. Mannosylerythritol lipids: production and applications. $J$. Oleo Sci. 64, 133-141 (2015).

5) Kitamoto, D.; Isoda, H.; Nakahara, T. Functions and potential applications of glycolipid biosurfactants. $J$. Biosci. Bioeng. 94, 187-201 (2002).

6) Kitamoto, D.; Akiba, S.; Hioki, C.; Tabuchi, T. Extracellular accumulation of mannosylerythritol lipids by a strain of Candida antarctica. Agric. Biol. Chem. 54, 31-36 (1990).

7) Morita, T.; Takashima, M.; Fukuoka, T.; Konishi, M.; Imura, T.; Kitamoto, D. Isolation of basidiomycetous yeast Pseudozyma tsukubaensis and production of glycolipid biosurfactant, a diastereomer type of mannosylerythritol lipid-B. Appl. Microbiol. Biotechnol. 88, 679-688 (2010).

8) Morita, T., Fukuoka, T., Imura, T., Hirose, N., Kitamoto, D. Isolation and screening of glycolipid biosurfactant producers from sugarcane. Biosci. Biotechnol. Biochem. 76, 1788-1791 (2012).

9) Konishi, M.; Morita, T.; Fukuoka, T.; Imura, T.; Kakugawa, K.; Kitamoto, D. Production of different types of mannosylerythritol lipids as biosurfactants by the newly isolated yeast strains belonging to the genus Pseudozyma. Appl. Microbiol. Biotechnol. 75, 521531 (2007).

10) Fukuoka, T.; Morita, T.; Konishi, M.; Imura, T.; Kitamoto, D. Structural characterization and surface-active properties of a new glycolipid biosurfactant, mono-acylated mannosylerythritol lipid, produced from glucose by Pseudozyma antarctica. Appl. Microbiol. Biotechnol. 76, 801-810(2007).

11) Fukuoka, T., Morita, T., Konishi, M., Imura, T., Kitamoto, D. Characterization of new glycolipid biosurfactants, tri-acylated mannosylerythritol lipids, produced by Pseudozyma yeasts. Biotechnol. Lett. 29, 11111118(2007).

12) Morita, T.; Fukuoka, T.; Imura, T.; Kitamoto, D. Accumulation of cellobiose lipids under nitrogen-limiting conditions by two ustilaginomycetous yeasts, Pseudozyma aphidis and Pseudozyma hubeiensis. FEMS Yeast Res. 13, 44-49 (2013).

13) Imura, T.; Kawamura, D.; Morita, T.; Sato, S.; Fukuoka, T.; Yamagata, Y.; Takahashi, M.; Wada, K.; Kitamoto, D. Production of sophorolipids from non-edible jatropha oil by Stamerella bombicola NBRC 10243 and evaluation of their interfacial properties. J. Oleo Sci. 62, 857-864 (2013).

14) Hewald, S.; Josephs, K.; Bolker, M. Genetic analysis of biosurfactant production in Ustilago maydis. Appl. Environ. Microbiol. 71, 3033-3040(2005).

15) Thompson, J.D.; Higgins, D.G.; Gibson, T.J. CLUSTAL $\mathrm{W}$ : improving the sensitivity of progressive multiple sequence alignment through sequence weighting, position-specific gap penalties and weight matrix choice. Nucleic Acids Res. 22, 4673-4680 (1994).

16) Morita, T.; Konishi, M.; Fukuoka, T.; Imura, T.; Kitamoto, H.K.; Kitamoto, D. Characterization of the genus Pseudozyma by the formation of glycolipid biosurfactants mannosylerythritol lipids. FEMS Yeast Res. 7, 286-292(2007). 